\title{
Influence of diversion angle on water and sediment flow into diversion channel
}

\begin{abstract}
Accumulation of the sediment in the stream of the diversion channels adversely affects its operational systems. Diversion channels are often constructed perpendicular to the main river. In this study, the water flow and sediment transport in the diversion channel with different angles were investigated in an attempt to maximize water discharge and minimize sediment discharge. A physical model with movable bed was used to simulate water and sediment flow with five diversion angles $(\theta)$ between $\left(30^{\circ}-90^{\circ}\right)$. Moreover, three bed width ratios $(\mathrm{Br})$ (the relation between diversion to main channel bed width) between $30 \%$ and $50 \%$ and five total discharges between $(7.25 \mathrm{~L} / \mathrm{s}$ to $12.25 \mathrm{~L} / \mathrm{s})$ were considered for each case of $(\theta)$. The results showed, up to $10 \%$, increasing in proportion discharge ratios for 30 and 45 diversion angles compared with $90^{\circ}$ diversion angle. The results also showed that the lowest diversion sediment concentration was provided by the $(\theta)$ of $30^{\circ}$. Across all scenarios, the average proportion concentration reduction was $64 \%$, compared with $90^{\circ}$ diversion angle. Closer observation of the diversion system mechanism confirmed that decreased $(\theta)$ result in decreased sediment concentrations in the diversion channels. In conclusion, the diversion channel water and sediment discharge could be effectively managed by changing the $(\theta)$ to $30^{\circ}$ or $45^{\circ}$ instead of $90^{\circ}$.
\end{abstract}

Keyword: Diversion channel; Diversion angle; Lowest diversion sediment concentration; Bed width ratio; Sediment concentration 